\title{
Scaling Calibration in the ATRACT Algorithm
}

\author{
Yan Xia ${ }^{1}$, Andreas Maier ${ }^{1}$, Frank Dennerlein ${ }^{2}$, Hannes G. Hofmann ${ }^{1}$, \\ Joachim Hornegger ${ }^{1,3}$ \\ ${ }^{1}$ Pattern Recognition Lab (LME), Friedrich-Alexander-University \\ Erlangen-Nuremberg, Erlangen, Germany \\ ${ }^{2}$ Healthcare Sector, Siemens AG, Erlangen, Germany \\ ${ }^{3}$ Erlangen Graduate School in Advanced Optical Technologies (SAOT), \\ Friedrich-Alexander-University Erlangen-Nuremberg, Erlangen, Germany \\ yan.xia@cs.fau.de
}

\begin{abstract}
Recently, a reconstruction algorithm for region of interest (ROI) imaging in C-arm CT was published, named Approximated Truncation Robust Algorithm for Computed Tomography (ATRACT). Even in presence of severe data truncation, it is able to reconstruct images without the use of any explicit extrapolation or prior knowledge. However, this method suffers from a scaling artifact in the reconstruction. In this paper, we have investigated a calibration applied in the projection domain to compensate this scaling problem. The proposed correction method is evaluated by using six clinical datasets in presence of different artificial truncation. The results show that a relative root mean square error (rRMSE) of up to $0.9 \%$ is achieved by the corrected ATRACT method.
\end{abstract}

\section{Introduction}

For three-dimensional (3D) X-ray imaging during the interventions, changes of the examined patient are often restricted to a small part of the field of view (FOV), e.g. cochlear implants, and needle biopsies. This suggests region of interest (ROI) imaging by irradiating the diagnostic interest area only. However, the corresponding 3D ROI reconstruction from laterally truncated projections poses a challenge to the conventional tomographic reconstruction algorithms and can result in a noticeable degradation of image quality.

So far various truncation correction methods have been proposed to overcome the effect of truncation artifact $[1,2,3,4]$. Recently, a novel method for ROI reconstruction of highly truncated projection data with neither the use of prior knowledge nor any explicit extrapolation has been suggested [5,6]. This method (ATRACT) is based on a decomposition of the standard ramp filter within FDK (Feldkamp, Davis, and Kress algorithm [7]) into a local and a non-local filtering step, where the local step is a 2D Laplace operator and the non-local step is a 2D Radon-based filtering that can be converted into a $2 \mathrm{D}$ convolution-based filtering [8]. The ATRACT method can provide satisfactory reconstruction results even in presence of severe data truncation. But it suffers from a global scaling 
and bias problem in the reconstruction. It is therefore our goal to compensate this problem in the projection domain by searching projection calibration parameters.

\section{Materials and Methods}

The ATRACT algorithm discussed here is an optimized version, where the nonlocal operation corresponds a 2D convolution-based filter. This significantly increases computational performance compared to Radon-based filtering [8].

\subsection{ATRACT Algorithm}

We focus on the circular cone-beam imaging geometry shown in Fig. 1. Then, the ATRACT algorithm can be written as follows:

- Step 1: Cosine- and Parker-like weighting of projection data to obtain $g_{1}(\lambda, u, v)$ :

$$
g_{1}(\lambda, u, v)=\frac{D m(\lambda, u)}{\sqrt{D^{2}+u^{2}+v^{2}}} g(\lambda, u, v)
$$

- Step 2: 2D Laplace filtering to obtain projection data $g_{2}(\lambda, u, v)$ :

$$
g_{2}(\lambda, u, v)=\left(\frac{\partial^{2}}{\partial u^{2}}+\frac{\partial^{2}}{\partial v^{2}}\right) g_{1}(\lambda, u, v)
$$

- Step 3: 2D convolution-based filtering to get filtered projection data $g_{F}(\lambda, u, v)$ :

$$
g_{F}(\lambda, u, v)=\frac{1}{4 \pi^{2}} \frac{R}{D} \int_{u_{1}}^{u_{2}} \int_{v_{1}}^{v_{2}} g_{2}\left(\lambda, u-u^{\prime}, v-v^{\prime}\right) \frac{\left|v^{\prime}\right|}{u^{\prime 2}+v^{\prime 2}} d u^{\prime} d v^{\prime}
$$
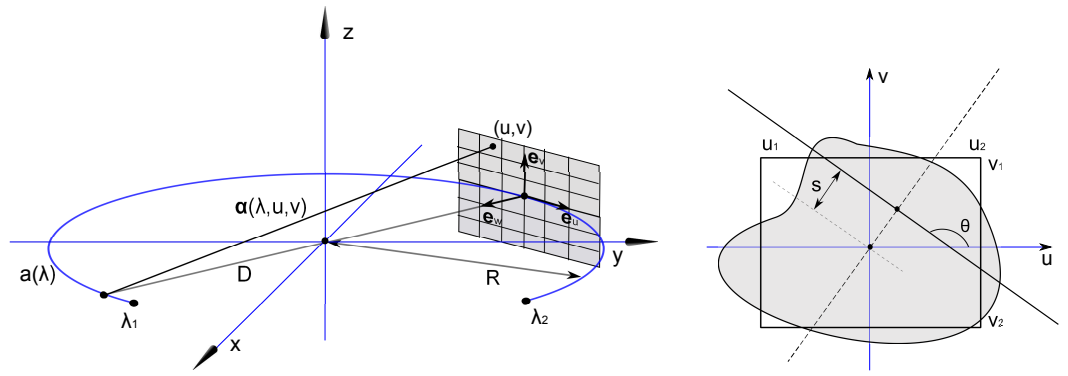

Fig. 1. Cone-beam geometry and associated notation: The curve $\mathbf{a}(\lambda)=$ $(R \cos \lambda, R \sin \lambda, 0)$ describes the trajectory of the $\mathrm{X}$-ray source, with the scan radius $\mathrm{R}$ and the rotation angle $\lambda$. The planar detector is parallel to the unit vectors $\mathbf{e}_{\mathbf{u}}(\lambda)$ and $\mathbf{e}_{\mathbf{v}}(\lambda)$ and at distance D from the source. $\mathbf{e}_{\mathbf{w}}(\lambda)$ is the detector normal. $(\theta, s)$ represent Radon-based coordinates. We use the function $g(\lambda, u, v)$ to describe the projection data at the point $(u, v)$ acquired at angle $\lambda$. 
- Step 4: 3D cone-beam backprojection to get $f^{(A T R A C T)}(x, y, z)$ :

$$
f^{(A T R A C T)}(x, y, z)=\int_{\lambda_{1}}^{\lambda_{2}} \frac{R D}{\left[R-(x, y, z) \cdot \mathbf{e}_{w}(\lambda)\right]^{2}} g_{F}(\lambda, u, v) d \lambda
$$

\subsection{Scaling Correction in ATRACT}

In the ATRACT algorithm, we remove the singularities at the edges of lateral data truncation after Laplace filtering. This causes a loss of the information on the thickness of the object. The following residual filtering of truncated projections will result in a offset with respect to the FDK filtering of non-truncated projection, as illustrated in Fig. 2(a). In the previous literature, a correction of scaling and bias was manually performed in the final reconstructed volume for each dataset, to align the value range between the reference and ATRACT.

In this paper, we compensate the scaling problem by calibrating the projectionrelated parameters. The scheme is formulated as follows:

$$
\begin{gathered}
g_{F}^{\text {corrected }}(\lambda, u, v)=g_{F}(\lambda, u, v)+\operatorname{off} \operatorname{set}(\lambda) \\
\operatorname{offset}(\lambda)=A \cdot \sum_{u_{1}}^{u_{2}} \sum_{v_{1}}^{v_{2}} g(\lambda, u, v)+B+C \cdot\left(u_{2}-u_{1}\right) \cdot\left(v_{2}-v_{1}\right),
\end{gathered}
$$

where $g_{F}(\lambda, u, v)$ and $g_{F}^{\text {corrected }}(\lambda, u, v)$ denote the filtered projections by ATRA$\mathrm{CT}$ without and with the scaling correction.

The attenuation-related linear parameters $A$ and $B$ were determined by comparing the difference between the filtered projections by ATRACT and by the full FOV FDK. The last term in Eqn. (6) is a compensation factor in case that truncation size is too small. The corresponding parameter $C$ was determined in the small truncation case. Fig. 2(b) shows the line profiles of ATRACT filtering of the truncated projection with and without correction as well as FDK filtering

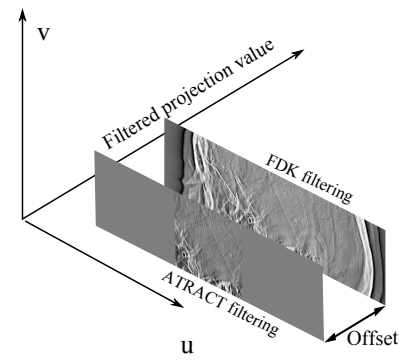

(a)

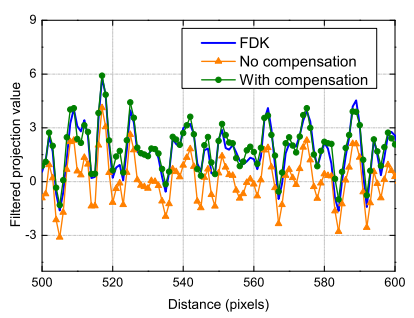

(b)

Fig. 2. (a) Offset problem in ATRACT filtering of a truncated projection. (b) Line profiles of the filtered projection by the corrected and uncorrected ATRACT and by FDK. 
of the truncation-free projection. Note that in the following evaluation parameters $A, B$ and $C$ are fixed in all datasets and calibration has to be performed only once for a given acquisition scenario.

\subsection{Experiment Setup}

To validate and evaluate the new scaling correction, six clinical datasets of patient heads were employed. All datasets were acquired on a C-arm system (Artis Zee, Siemens AG) from St. Luke's Episcopal Hospital (Houston, TX, USA). All scans containing 496 projection images $(1240 \times 960)$ with the resolution of 0.308 $\mathrm{mm} /$ pixel were acquired on a $200^{\circ}$ short-scan circular trajectory. The calibration parameters were determined using the dataset 1 with $A=-3.68 E-7$, $B=1.78$ and $C=-6.76 E-7$.

Three experiment setups were considered. In Setup 1, no collimation was applied, yielding the non-truncated projection data. In Setup 2 and 3, the data sets were virtually collimated (by setting the outside to zero) to the two different levels (FOV: $104 \mathrm{~mm}$ and $72 \mathrm{~mm}$ ), so that only the desired FOV was kept.

All clinical data were reconstructed onto a Cartesian grid $(512 \times 512 \times 350)$ with sampling spacing $\triangle x=\triangle y=\triangle z=0.4 \mathrm{~mm}$ in Setup 1 and 2 and with different sampling spacing $\triangle x=\triangle y=\triangle z=0.2 \mathrm{~mm}$ in Setup 3. The standard FDK reconstruction of Setup 1, i.e. non-truncated projection was used as the reference in each clinical case. The truncated datasets were reconstructed by the corrected and uncorrected ATRACT algorithm. The quantitative evaluation for the six clinical datasets was carried out by using the relative root mean squared error (rRMSE) and the correlation coefficient within the entire ROIs.

\section{Results}

The reconstruction results of the clinical dataset 1 and 4 from all three setups are presented in Fig. 3 and Fig. 4, respectively. In the visual inspection, no significant difference is observed in the same grayscale window between the corrected ATRACT-based ROI reconstructions and the reference reconstructions from non-truncated data, even in presence of different truncation levels. However, for visualizing the reconstructions from the uncorrected ATRACT, totally different display windows are applied. Also note that the incorrect scaling and bias in the reconstructed volume might cause difficulties for any volume-based post-processing algorithm in different stages of the imaging pipeline. Here, for instance, we can observe that the ring artifact reduction algorithm does not produce proper results (marked by the arrows) if no correction is applied.

A summary of the quantitative evaluation from the reconstructions of all six clinical datasets are shown in Table 1 . The corrected ATRACT reduces the rRMSE of up to $0.92 \%$ compared to the rRMSE of $3.29 \%$ for uncorrected one. Also, it is clear that the new scaling calibration performs nicely in all evaluated datasets with the same calibration parameters $A, B$ and $C$. Note that due to the fact that the correlation coefficient is independent to the scaling and bias 
problem in the reconstruction, no significant difference is observed for these values between the corrected ATRACT and the uncorrected one. Improvements emerge only from the improved ring correction.
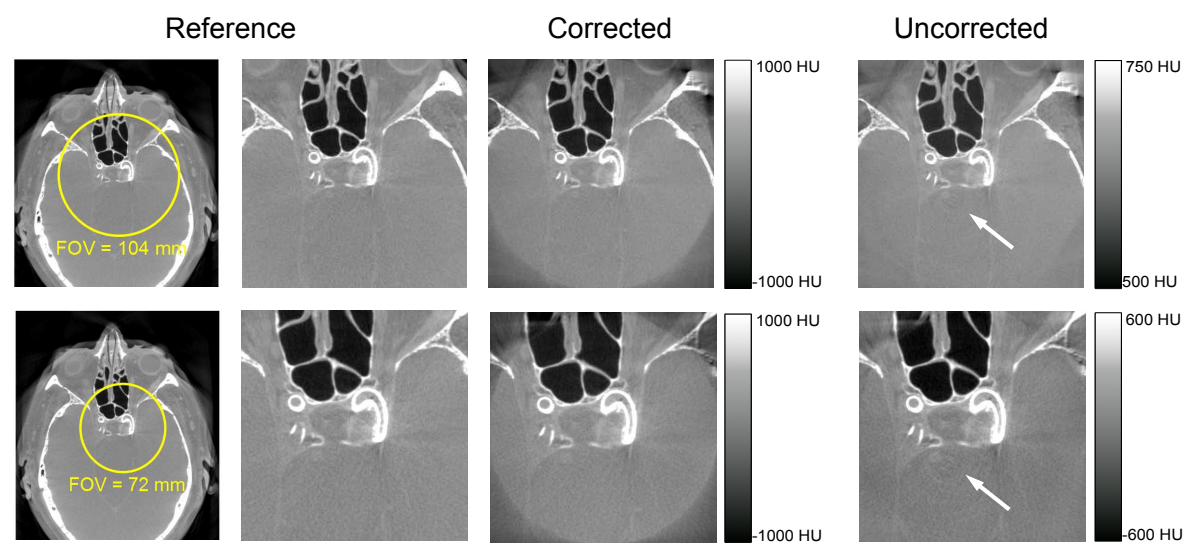

Fig. 3. Reconstruction results of the clinical dataset 1 by the ATRACT algorithm. From left to right: The FDK reconstructions of non-truncated data, zoomed FDK reconstructions, the corrected ATRACT reconstructions, the uncorrected ones.

\section{Discussion}

In this paper we presented a calibration that can be applied on an existing truncation correction using ATRACT. The average RMSE was reduced by 7 times using the corrected ATRACT method. A potential limitation of this study is

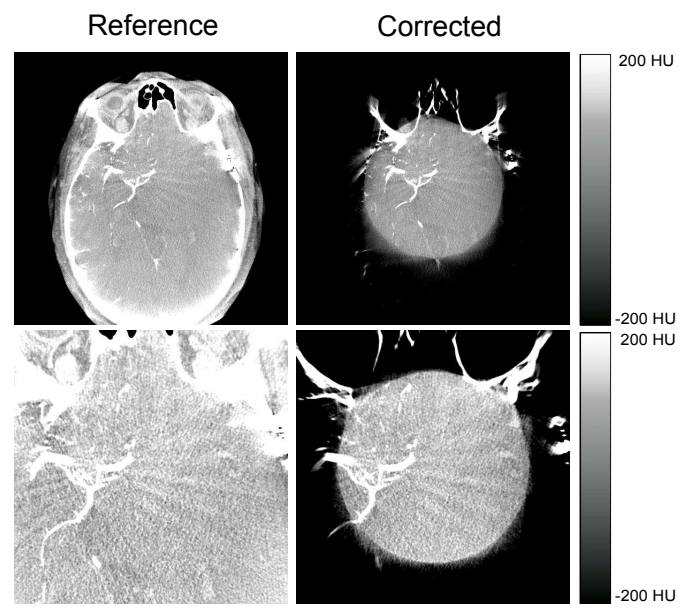

Fig. 4. Homogeneous area of the clinical dataset 4 reconstructed by the corrected ATRACT algorithm in a compressed display window (C:0 $\mathrm{HU}, \mathrm{W}: 200 \mathrm{HU})$. The first row: the reference with slice thickness $0.4 \mathrm{~mm}$, the ROI reconstruction by the corrected ATRACT from Setup 2 (FOV:104mm). The bottom row: the reference with slice thickness 0.2 $\mathrm{mm}$, the ROI reconstruction by the corrected ATRACT from Setup 3 (FOV:72mm). 
Table 1. Summary of the quantitative evaluation computed from the reconstructions of the six patient head datasets by means of the corrected and uncorrected ATRACT.

\begin{tabular}{llllll}
\hline & & \multicolumn{2}{c}{ rRMSE (\%) } & \multicolumn{2}{c}{ Correlation } \\
\cline { 3 - 6 } Dataset FOV $(\mathrm{mm})$ & \multicolumn{2}{c}{ Uncorrected Corrected } & Uncorrected Corrected \\
\hline 1 & 104 & 7.56 & 0.922 & 0.989 & 0.990 \\
& 72 & 3.29 & 0.923 & 0.981 & 0.982 \\
2 & 104 & 10.4 & 1.72 & 0.984 & 0.984 \\
& 72 & 9.58 & 1.49 & 0.990 & 0.992 \\
3 & 104 & 7.72 & 1.55 & 0.974 & 0.976 \\
& 72 & 11.4 & 2.54 & 0.970 & 0.970 \\
4 & 104 & 9.78 & 2.10 & 0.985 & 0.985 \\
& 72 & 12.6 & 1.99 & 0.990 & 0.991 \\
5 & 104 & 20.6 & 1.36 & 0.951 & 0.953 \\
& 72 & 22.0 & 2.04 & 0.982 & 0.989 \\
6 & 104 & 15.5 & 1.48 & 0.985 & 0.985 \\
& 72 & 15.7 & 1.53 & 0.994 & 0.994 \\
\hline
\end{tabular}

that only patient head datasets were employed. Differences between the anatomy of the patient are likely to result in variations in calibration parameters.

\section{References}

1. J Hsieh, E Chao, J Thibault, B Grekowicz, A Horst, S McOlash, and T J Myers. A novel reconstruction algorithm to extend the CT scan field-of-view. Med Phys, 31(9):2385-2391, 2004.

2. M Defrise, F Noo, R Clackdoyle, and H Kudo. Truncated hilbert transform and image reconstruction from limited tomographic data. Inverse Probl, 22(3):10371053, 2006.

3. D Kolditz, Y Kyriakou, and W A Kalender. Volume-of-interest(VOI) imaging in Carm flat-detector CT for high image quality at reduced dose. Med Phys, 37(6):27192730, 2010.

4. A Maier, B Scholz, and F Dennerlein. Optimization-based Extrapolation for Truncation Correction. In 2nd CT Meeting, pages 390-394, 2012.

5. F Dennerlein. Cone-beam ROI reconstruction using the Laplace operator. In Proc Fully 3D 2011, pages 80-83, 2011.

6. F Dennerlein and A Maier. Region-of-interest reconstruction on medical C-arms with the ATRACT algorithm. In Proc SPIE, page 83131B, 2012.

7. L A Feldkamp, L C Davis, and J W Kress. Practical cone beam algorithm. J Opt Soc Am, 1(6):612-619, 1984.

8. Y Xia, A Maier, F Dennerlein, H G Hofmann, and J Hornegger. Efficient 2D filtering for cone-beam VOI reconstruction. In Proc IEEE NSS/MIC, 2012. 2415-2420. 